\title{
PlayGame: A Platform for Diagnostic Games
}

\author{
$\operatorname{Li} \operatorname{Tan}^{\star}$
}

Department of Computer and Information Science, University of Pennsylvania Philadelphia, PA 19104, USA

tanli@saul.cis. upenn. edu

\begin{abstract}
We introduce an integrated tool for implementing and playing various diagnostic games. The tool uses a semantics hierarchy introduced in [6] to improve code sharing among various diagnostic games and reduce the cost of introducing a new game. PlayGame synthesizes the winning strategy using the evidence that is an abstract and uniform encoding of the proof computed by a checker, and hence instead of relying on any particular checker the tool works on a variety of checkers that can be extended to produce such evidence. PlayGame implements a $\mu$-calculus game and a full range of equivalence/preorder games on the Concurrency Workbench-New Century (CWB-NC).
\end{abstract}

\section{Introduction}

Games have been used in the verification community to model verification problems, to seek better solutions, and to understand verification results. The early work by Stirling [5] on bisimulation games and $\mu$-calculus games unveils the potential of such games as diagnostic routines. In a diagnostic game the user competes with the computer to show that the verification result is incorrect. By losing each and every play to the computer the user shall be then convinced of the correctness of the verification result. A diagnostic game can provide valuable diagnostic information in an interactive way that a traditional diagnostic routine such as counterexample mechanism cannot. Individual efforts have been made to implement certain types of diagnostic games. The recent release of Edinburgh Concurrency Workbench [1] includes the support for a $\mu$-calculus game and a strong bisimulation game. The verification tool Truth [3] also implements a $\mu$ calculus game. These tools are designed to use some specific checkers, mostly game-based checkers, to build the winning strategy for the computer. It is left to see how diagnostic games can be built on top of other existing checkers. Another problem in diagnostic games is that with so many verification semantics, each of which requires different rules for game, defining and implementing them separately is a daunting task. We introduce PlayGame, a tool that provides a consistent interface for implementing diagnostic games and incorporating checkers. Figure 1 shows the architecture of the tool.

\footnotetext{
* Research supported by NSF grants CCR-9988489 and CCR-0098037, Army Research Office grants DAAD190110003, DAAD190110019, and DAAD190110473.
} 


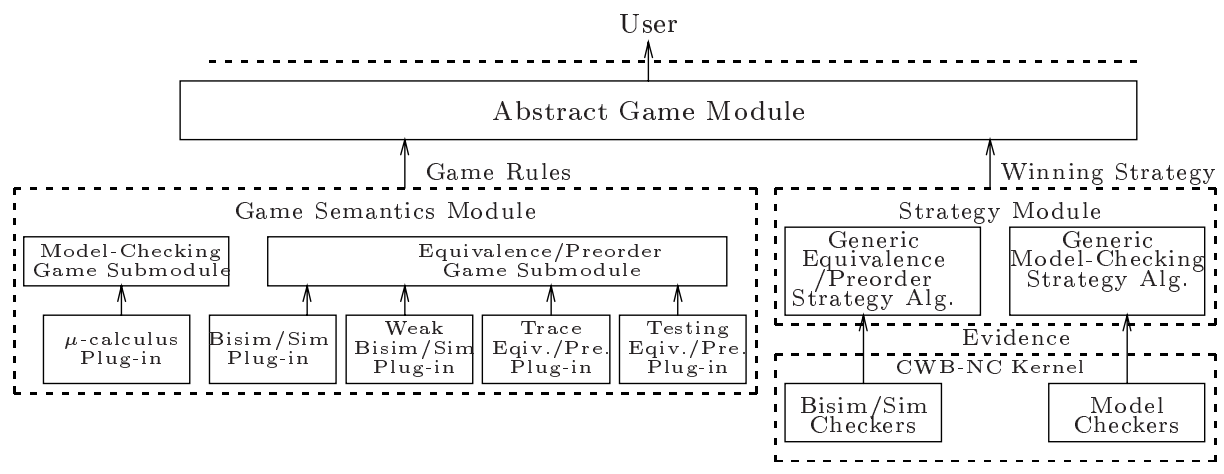

Fig. 1. The architecture of PlayGame

The tool is designed to use the evidence that is an abstract and uniform encoding of the proof constructed by a checker during verification. Its precise definition is given in our previous work $[7,8]$. Instead of relying on a particular checker, the tool works on a variety of existing checkers that can be extended to produce such evidence, as discussed in $[7,8]$. To support different verification semantics, we introduce a semantics hierarchy [6] that abstracts game rules to different layers. To introduce a new game, one only needs to provide the semantic layers unique to the game. PlayGame implements a $\mu$-calculus model-checking game and eight different equivalence/preorder games including strong bisimulation/simulation games, weak bisimulation/simulation games, trace equivalence/preorder games, and testing equivalence/preorder games that cover all the equivalence/preorder semantics supported by Concurrency Workbench - New Century (CWB-NC)[2]. PlayGame also provides a consistent user interface for all the games that reduces the time required to learn a new game.

\section{PlayGame}

\subsection{Designing PlayGame}

The design of PlayGame reflects the game semantics hierarchy defined in [6]. The abstract game module implements an abstract version of games and the features common to all games. The game semantics module defines the rules for each individual game. The strategy module synthesizes winning strategies from the evidences submitted by checkers.

Abstract Game Module. A typical verification game has two players: player I, who insists a negative verification result, and player II, who believes otherwise. Each game has its own rules that must be determined in the following sense: if the correct answer to the verification problem is negative, then I has a strategy to win each and every play no matter how II moves; otherwise, II shall have a winning strategy. When a game is used as a diagnostic routine, it involves two 
sides: the computer vs. the user. The computer assumes the role of a player in favor of the verification result. Thus, by losing each and every play to the computer, the user is convinced of the verification result. The abstract game module implements the aforementioned abstract version of games. It also introduces the role of a referee that enforces the rules supplied by the game semantics module. It also implements the common functions including the bookkeeping and the user interface.

Game Semantics Module. Game semantics module defines the rules for each game. For the games studied before such as $\mu$-calculus games and strong bisimulation games [5], our definition is close to previous results but also takes into account the human factor. Our revision intends to keep plays concise and informative. For example, the definition of a $\mu$-calculus game by Stirling [5] requires two steps and the participant of a player to unroll a fixpoint expression $(\mu z . \Psi$ or $\nu z . \Psi)$, while the choice of the player unrolling the expression is really irrelevant. In the revised rules, it takes only one step and becomes part of the referee's job. For those games that to the best of our knowledge have not been defined in literature such as testing equivalence/preorder games, we define the games based on the target verification semantics. To further improve code sharing we exploit similarity among each category of games. For instance, in [6] we introduce a generalized equivalence game submodule and semantics plug-ins. To introduce an equivalence game, one only needs to supply a relatively small plug-in that specifies part of rules unique to this game. In our experience abstracting rules to different layers saves about 70-80\% coding work when introducing a new game.

Strategy Module. Synthesizing a winning strategy for the computer is the key to diagnostic games. Traditionally winning strategies are constructed by a game-based checker [4]. In PlayGame the strategy is constructed from the evidence supplied by a checker. In $[7,8]$ we propose uniformly-encoded evidences for various verification semantics: for equivalence checking the evidence is a partition refinement tree; for preorder checking it is a kernel-auxiliary partition refinement tree [7], a variant of partition refinement tree in which each node contains a set of upper states (auxiliary set) in addition to a set of lower (and equivalent) states (kernel set); for model checking the evidence is a support set [8]. It turns out that winning strategies for the games of the same category are quite similar. For equivalence games, the strategy is to keep the states of two processes in different leaves of the partition refinement tree, and hence the algorithm for synthesizing the winning strategy from the evidence is implemented per category basis.

\subsection{Using PlayGame}

PlayGame is implemented on the CWB-NC. To activate a game, the user simply issues a verification command with a special flag. The CWB-NC with PlayGame calls a checker and enters the interactive game mode after the verification ends. 
A play starts with the referee declaring the roles of the computer and the user based on the verification result, then it proceeds by rounds. Figure 2 shows a sample round in a weak bisimulation game. The look and feel of other games are quite similar. The referee judges the winner with an explanation. If no one wins yet, he decides how a round shall proceed. The user is prompted for his/her options of the next move. The user can also choose to take back a few steps.

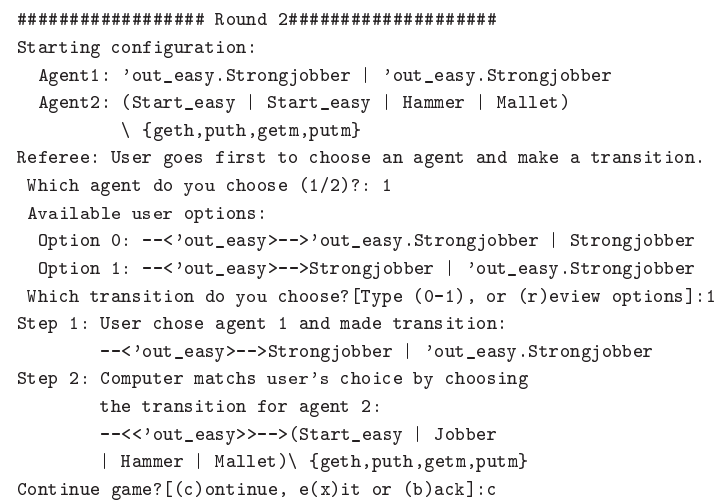

Fig. 2. A sample round

\section{Conclusions and Future Work}

We introduce PlayGame, an integrated platform for diagnostic games. Two novel features in its design are the use of semantics hierarchy, which enables code sharing among different games, and the building of winning strategy using checkerindependent evidences, which makes it easier to incorporate new checkers. It implements a $\mu$-calculus game and the full range of equivalence/preorder games. In future we want to study and implement the diagnostic game for other logics.

\section{Acknowledgments}

The author would like to thank Rance Cleaveland for many interesting and fruitful discussions, and Madhusudan Parthasarathy for reviewing the draft of this paper.

\section{References}

1. The Edinburgh Concurrency Workbench. The University of Edinburgh, 1999.

2. R. Cleaveland and S. Sims. The NCSU concurrency workbench. In Proceedings of CAV'96, volume 1102 of $L N C S, 1996$.

3. M. Leucker and T. Noll. Truth/SLC - A parallel verification platform for concurrent systems. In Proceedings of CAV'01, volume 2102 of LNCS, 2001.

4. P. Stevens and C. Stirling. Practical model-checking using games. In Proceedings of TACAS '98, volume 1384 of LNCS, 1998.

5. C. Stirling. Games and modal mu-calculus. In Proceedings of TACAS'96, volume 1055 of $L N C S, 1996$.

6. L. Tan. An abstract schema for equivalence games. In Proceedings of VMCAI'02, volume 2294 of $L N C S, 2002$.

7. L. Tan. Evidence-based Verification. PhD thesis, State University of New York at Stony Brook, May 2002.

8. L. Tan and R. Cleaveland. Evidence-based model checking. In Proceedings of CAV'02, volume 2404 of LNCS, 2002. 